\title{
Stimulated electronic Raman scattering in Cs vapour: a simple tunable laser system for the 2.7 to $3.5 \mu \mathrm{m}$ region
}

\author{
D. COTTER, D. C. HANNA \\ Department of Electronics, University of Southampton, Southampton SO9 $5 \mathrm{NH}, \mathrm{UK}$
}

Received 5 May 1977

\begin{abstract}
Stimulated electronic Raman scattering (SERS) in atomic vapours provides a simple method of extending the tuning ranges of pulsed dye lasers well into the infrared region. The special advantages of this technique in comparison with other types of tunable infrared lasers are discussed, and are illustrated by describing a SERS system which uses a modest nitrogen laser-pumped dye laser ( $20 \mathrm{~kW})$. This produces infrared radiation tunable from 2.67 to $3.47 \mu \mathrm{m}$ by SERS in caesium vapour, which is contained in a heat pipe oven. Photon conversion efficiencies of up to $50 \%$ are obtained. The design of the heat pipe oven, operation of the system and optimization of experimental parameters are described in detail.
\end{abstract}

\section{Introduction}

Within the last 10 years, the availability of tunable coherent light sources $[1,2]$ has made possible a number of new observations and techniques in atomic and molecular spectroscopy. These new possibilities stem from the high power, spectral brightness, and directionality of the output from these sources, and in some cases, from a capability for short time-resolution. In many fields of application the impact of these devices is only beginning to be felt.

The most widely used and best developed of these sources is the dye laser, which is tunable throughout the visible region of the spectrum from the near ultraviolet to near infrared (approximately $300 \mathrm{~nm}-1 \mu \mathrm{m}$ ) [3]. Attempts to extend dye laser operation to longer wavelengths have not met with much success. This is an unfortunate limitation of dye lasers since the infrared region is of particular importance in molecular spectroscopy. A number of different types of tunable infrared laser now exist at various stages of development $[1,2]$ and these are leading to many new applications such as remote detection of atmospheric pollutants, nonlinear molecular spectroscopy, molecular super-excitation phenomena, photochemistry and isotope separation. Unfortu- nately most of these tunable infrared lasers have disadvantages of complexity and high cost. However an exception to this is a recently developed method of infrared generation based on stimulated electronic Raman scattering (SERS) in atomic vapours [4-7]. This nonlinear optical process has been used to convert the output from pulsed dye lasers operating in the blue and near UV region into a tunable source of infrared radiation. For the many laboratories which are already equipped with suitable dye lasers, the SERS

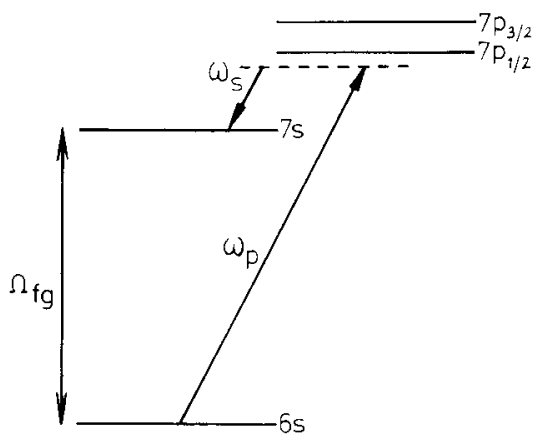

Figure 1 Energy level diagram of an alkali atom showing the SERS scheme for infrared generation. (The different levels have been given labels specific to $6 s \rightarrow 7 s$ scattering in caesium). 
method provides an exceptionally simple and cheap method of extending the wavelength coverage of these lasers into regions of the medium infrared range.

The basic SERS scheme is illustrated in the electronic energy level diagram of Fig. 1, where an alkali atomic system has been assumed. The intense dye laser (pump) light is used to excite a Raman transition between the electronic ground state and an excited $s$ state. In this way Raman shifted (Stokes) radiation is produced at the frequency $\omega_{s}=\omega_{\mathrm{p}}-\Omega_{\mathrm{fg}}$, where $\omega_{\mathrm{p}}$ is the pump frequency and $\hbar \Omega_{\mathbf{f g}}$ is the energy of the electronic Raman transition [8]. For SERS in alkali metal vapours this Raman shift is large $\left(20000-30000 \mathrm{~cm}^{-1}\right)$ allowing infrared Stokes wavelengths to be generated directly from a visible or near UV dye laser output. Since the Raman shift is constant, as the pump frequency is tuned the generated Stokes frequency will follow and so SERS provides a method for producing a tunable infrared output. Furthermore this Raman generation process can be made efficient by resonance enhancement. This is obtained by tuning $\omega_{\mathrm{p}}$ close to a singlephoton resonance between the ground state and the doublet $p$ levels. This produces a large Raman gain for Stokes scattering to the final $s$ level having the same principal quantum number as the intermediate $p$ level [6].

Experimentally the SERS method is very straightforward; the pulsed dye laser beam is simply focussed into a heated cell containing the alkali vapour, and the infrared radiation emerges in a narrow collimated beam collinearly with the incident laser beam. The only components that are required in addition to the pulsed dye laser system are the vapour cell and an optical filter to discriminate between the SERS output and other radiation emerging from the atomic vapour.

We describe here an infrared source based on SERS in caesium vapour which is pumped by a dye laser of modest peak power $(\sim 20 \mathrm{~kW})$. In this particular case the $6 s \rightarrow 7 s$ SERS transition (Raman shift $\Omega_{\mathrm{fg}}=18536 \mathrm{~cm}^{-1}$ ) is used to produce infrared radiation tunable over the wavelength range $2.7-3.5 \mu \mathrm{m}$ and with an output power of up to $1 \mathrm{~kW}$ over a significant part of the tuning range. The aim of this paper is to provide details of the practical aspects of the construction and operation of the source and the optimization of experimental parameters. We also wish to indicate the special advantages, as well as some disadvantages, of this technique for infrared generation compared with other methods.

\section{Vapour cell}

The caesium vapour, at pressures of $1-30$ torr, is contained in a heat pipe oven. In such a cell the alkali metal is constantly recirculated by capillary action through a mesh 'wick' (see Fig. 2), thus allowing operation over long periods of time without the usual problems caused by the metal depositing out at the cold end windows. For a detailed explanation of the operating principles of heat pipe ovens, the reader is referred to a description by the inventors, Vidal and Cooper [9]. Fig. 2 shows an oven which we have found suitable for use with caesium vapour. The end windows are entirely separated from the vapour by an inert buffer gas (argon), and are kept at room temperature by means of the water cooling coils. In this way the windows can be attached to the ends of the oven body using ordinary rubber $O$-ring vacuum seals. In the present case, windows made of fused silica (Infrasil) have been used, although we have also used caesium iodide and calcium fluoride windows when generating longer infrared wavelengths [6]. Another special property of heat pipes is that very uniform vapour densities can be maintained over long distances, which may be necessary for optimum phasematching in parametric conversion processes such as third harmonic generation [10]. However in SERS there is no phase-matching condition to be

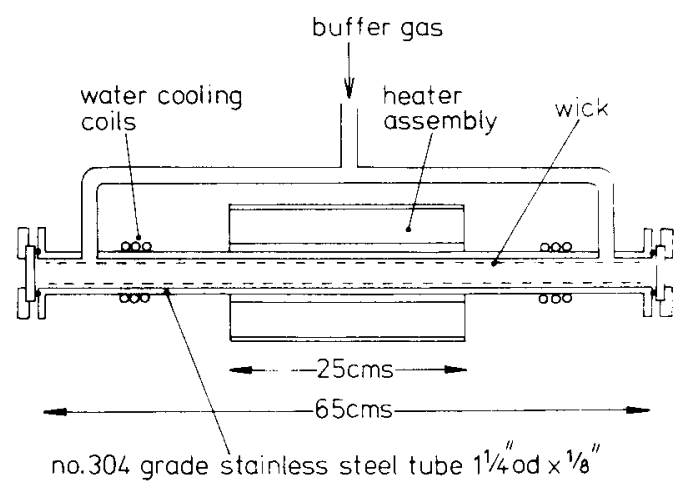

Figure 2 Heat pipe oven used to contain caesium vapour at pressures of 1-30 torr (see text for details). 
satisified, and this property of heat pipe ovens is not important.

In the oven shown in Fig. 2, there is a length of about $9 \mathrm{~cm}$ between the heated section and each of the copper coiling coils. In an earlier design, this length was much shorter, as little as $1-2 \mathrm{~cm}$, but this caused some difficulties as the caesium tended to solidify and accumulate in the cooled regions and was then prevented from flowing as a liquid back to the central heated region. As well as depleting the vapour, this accumulation of solid material was sufficient to partially obscure the light beams after a few weeks of operation. However, the later design appears to be free from this problem. An oven of this type, used with caesium at pressures of 1-30 torr, has given entirely trouble free operation and has been in use for more than 350 hours over a period of 8 months including more than 50 heating/cooling cycles. There have been no signs of contamination or fogging of the end windows by the alkali vapour.

The interior surface of the steel tube was carefully cleaned by scouring the inside with damp cotton wadding and carborundum powder. The tubing was then washed in water, rinsed in aqua regia, washed again and dried. The heat pipe wick consists of 5 or 6 turns of stainless steel mesh (100 mesh, grade 304 stainless steel plain weave gauze, $41 \mathrm{swg}$ ) and is a tight fit inside the steel tube extending the full length between the end windows. Before insertion the mesh was cleaned in a similar manner to that above. Finally it is, of course, necessary to ensure that the completed construction is vacuum tight in order to avoid gradual oxidation of the alkali metal.

The central $25 \mathrm{~cm}$ of the tube was wrapped with an electrical heating tape having a quartz fabric insulation [11]. Silica tape was used to hold the heater in place, and then over-wrapped with a layer $3-4 \mathrm{~cm}$ thick of ceramic fibre blanket [12] (See Fig. 2). Finally the whole heating assembly was secured with glass tape. With this construction about $60-90 \mathrm{~W}$ of electrical heating is sufficient for $1-30$ torr operation $\left(280-430^{\circ} \mathrm{C}\right)$ using caesium.

The oven is loaded with about $10 \mathrm{~g}$ of the alkali metal, which is normally obtained from the suppliers packed in a glass breakseal ampoule. Since caesium ignites and sputters violently in contact with air, it must be treated with some caution.
A convenient method of loading the metal is to fill the oven, yet unheated, with an inert buffer gas (for example argon) and maintain a small positive pressure so that with the end windows removed there is an outflow of gas. The glass ampoule, clean and intact, can be introduced into the tube and then, when positioned centrally, the tip of the ampoule can be broken (using a glass rod for example). The end windows are then replaced, the pressure of argon in the oven reduced to around 10 torr, and the oven heater switched on for about 2 hours in order to vapourize the caesium from the ampoule. When the oven has been allowed to cool again to room temperature, the argon pressure can be increased once more to slightly greater than atmospheric pressure before the end windows are taken off so that the used ampoule can be removed carefully.

The procedure for starting up the oven is to set the required pressure of argon and switch on the heater. With our particular arrangement about 1 hour is required for warm up. If the volume of the interconnecting vacuum pipes and gauges is reasonably large compared with the volume of the heated zone in the oven, then the equilibrium vapour pressure will be only slightly greater than the initial pressure of the cold buffer gas. Our usual practice has been to switch off the heat pipe at the end of each day of use and restart it again as required.

\section{Pump source}

The dye laser, which is of our own construction, is pumped by $200 \mathrm{~kW}$ of output from a Molectron UV-300 nitrogen laser. It is essentially similar to the well-known design by Hänsch [13], except that the beam-ending telescope has been replaced by a prism [14]. Tuning is by means of a holographic grating used in first order, and automatic wavelength scanning is achieved using a stepping motor drive to rotate the grating mount. Only one dye, a solution of 7-diethylamino-4-methyl coumarin in methanol, is required to cover 449 $467 \mathrm{~nm}$. This is the range of pump wavelengths for which this SERS source produces an infrared output using the $6 s \rightarrow 7 s$ Raman transition in caesium. With this dye an output energy of typically $140 \mu \mathrm{J}$ in a $7 \mathrm{~ns}$ pulse ( $20 \mathrm{~kW}$ peak power) is obtained from the dye laser. By taking a little care with the initial alignment of the laser, a diffraction- 
limited output beam is obtained with nearlyGaussian intensity profiles in the vertical and horizontal directions. The spectral width of the output obtained from this laser is $0.1 \mathrm{~cm}^{-1}$.

\section{SERS performance}

We have found that the best performance in terms of infrared tuning range and output energy is obtained with a caesium vapour pressure of 10 torr and with the dye laser focussed at the centre of the vapour cell such that the confocal parameter $b_{\mathrm{p}}$ is approximately equal to the length of the vapour column $L\left(L / b_{\mathrm{p}} \approx 1, L=25-30 \mathrm{~cm}\right)$. The SERS output energy obtained under these conditions is shown in Fig. 3 as a function of dye laser tuning. The highest output energies of $7-8 \mu \mathrm{J}$ represent photon conversion efficiencies of up to $50 \%$. (These measurements were made using a Laser Precision pyroelectric energy meter, and due allowance was given for the optical losses in the vapour cell windows and Ge filter.) Observations using a fast InAs detector indicate a SERS pulse length of $\sim 5 \mathrm{~ns}$, implying a peak output power of $\sim 1.5 \mathrm{~kW}$.

There are three distinct minima in the SERS output energy curve shown in Fig. 3. The outer two occur when the dye laser corresponds in frequency with the resonance doublet $6 s-7 p_{1 / 2,3 / 2}$, and are accompanied by weak transmission of the dye laser beam through the vapour. These minima are therefore probably due to single-photon absorption of the pump radiation as well as losses due to other resonantly enhanced multi-photon processes. The central minimum at $\sim 21855 \mathrm{~cm}^{-1}$ was not accompanied by any observable dip in transmission of the dye laser beam. Its origin is not known for certain, although we suspect that it may be due to a single-photon excited state absorption of the SERS radiation [15].

SERS output energies greater than $30 \mathrm{~nJ}$ $(\sim 10 \mathrm{~W})$ were obtained for a continuous range of pump frequencies between 21420 and $22270 \mathrm{~cm}^{-1}$ giving a tunable infrared output over the $860 \mathrm{~cm}^{-1}$ range $2.67-3.47 \mu \mathrm{m}$. This arbitrary criterion for defining the extent of the tuning range has been selected for two practical reasons. Firstly the detected energy of $50 \mathrm{~nJ}$ gives a signal-to-noise ratio of $\sim 100$ when using a typical room temperature pyroelectric detector. Secondly, beyond the tuning range indicated there is a large pulse-topulse variation in the output energy. Whereas at the centre of the tuning range the infrared output amplitude stability is $\sim \pm 5 \%$ (approximately the same as that of the dye laser), at $\sim 80 \mathrm{~cm}^{-1}$ from the edge of the tuning range (as defined above) the amplitude stability is $\sim \pm 20 \%$, and at the edge of the range it is $\sim \pm 80 \%$. At frequencies outside the defined tuning range not every dye laser pulse produces a detectable Raman output, until at $100 \mathrm{~cm}^{-1}$ beyond the edge of the range, only about $5 \%$ of the incident dye laser pulses produce an infrared output greater than $1 \mathrm{~nJ}(\sim 200 \mathrm{~mW})$.

The tunable SERS output is accompanied by a number of fixed frequency emissions, and for certain applications it may be necessary to remove these by filtering the output beam. Most of these emissions correspond in frequency to identifiable single-photon atomic transitions, and some are comparable in energy to the SERS itself. These include emissions at $1.36 \mu \mathrm{m}\left(7 p_{3 / 2}-5 d_{5 / 2}\right)$,

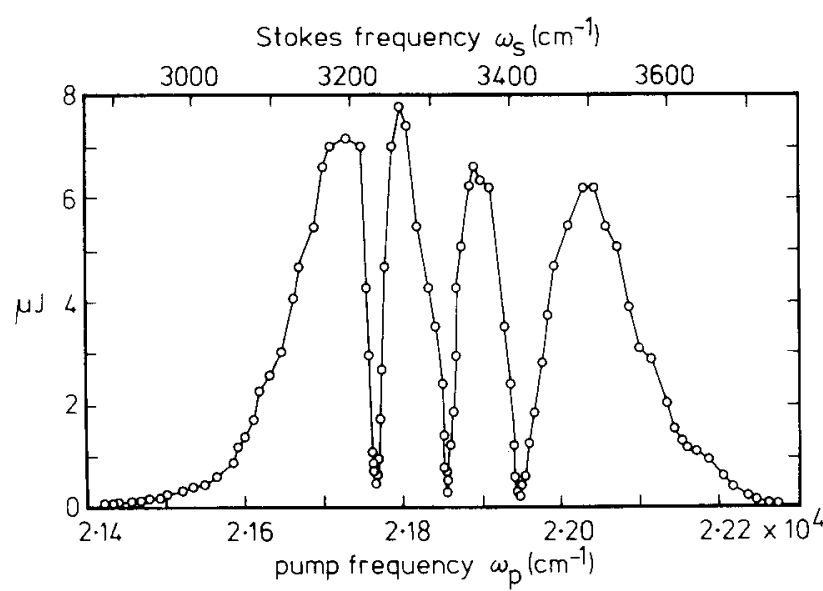

Figure 3 Infrared output energy per pulse as a function of dye laser tuning. 
$1.38 \mu \mathrm{m}\left(7 p_{1 / 2}-5 d_{3 / 2}\right), 1.36 \mu \mathrm{m}\left(7 s_{1 / 2}-6 p_{1 / 2}\right)$ and $1.47 \mu \mathrm{m}\left(7 s_{1 / 2}-6 p_{3 / 2}\right)$. Emissions at $3.01 \mu \mathrm{m}$ $\left(5 d_{3 / 2}-6 p_{1 / 2}\right)$ and $3.49 \mu \mathrm{m}\left(5 d_{5 / 2}-6 p_{3 / 2}\right)$ have only been observed for dye laser wavelengths longer than about $469 \mathrm{~nm}\left(21320 \mathrm{~cm}^{-1}\right)$, which is beyond the range of pump wavelengths for which a SERS output can be obtained. No emissions have been observed at $2.93 \mu \mathrm{m}\left(7 p_{3 / 2}-7 s_{1 / 2}\right)$ or $3.09 \mu \mathrm{m}\left(7 p_{1 / 2}-7 s_{1 / 2}\right)$. (An exception in this case may be when the dye laser is tuned to within $1-2 \mathrm{~cm}^{-1}$ of either of the $7 p_{1 / 2}$ and $7 p_{3 / 2}$ levels. in which case strong fixed frequency emissions, if present, would not be easily resolved from the SERS output [16]). This is very fortunate from the point of view of experimental convenience because it is thus possible to discriminate between the SERS signal and all the other radiation emerging from the vapour cell (including the residual dye laser beam) by simply filtering the output using an uncoated polished slice of germanium. It has been established that between the $1.8 \mu \mathrm{m} \mathrm{Ge}$ band edge and the $4.3 \mu \mathrm{m}$ cut-off wavelength of the 'Infrasil' cell window the SERS signal contributes more than $99 \%$ of the total energy detected after this Ge filter. Thus it is possible, in this case, to dispense with a monochromator or other tunable filter as the means of discriminating between the different emissions. This greatly simplifies the arrangement for wavelength scanning.

Fig. 4 shows the different SERS tuning curves (output energy versus dye laser frequency) that are obtained for different values of the focussing factor $L / b_{\mathrm{p}}$, where $b_{\mathrm{p}}$ is the confocal parameter of the dye laser beam focussed at the centre of the oven and $L$ is the length of the vapour column. For these measurements the vapour pressure was 10 torr. At first there is a rapid increase in the SERS tuning range as the pump focussing is made tighter (increasing $L / b_{\mathrm{p}}$ ). This is to be expected for two reasons. Firstly, the Raman gain and threshold depends on the pump intensity. For a focussed Gaussian pump beam the small signal gain is proportional to $\arctan \left(L / b_{\mathfrak{p}}\right)$ [17]. Secondly, there is significant absorption of the dye laser radiation by alkali dimers. At caesium vapour pressures of 1-30 torr the number of $\mathrm{Cs}_{2}$ molecules is $0.5-2 \%$ of the total number density. The absorption crosssection of these dimers is $\sim 3 \times 10^{-21} \mathrm{~m}^{2}$ for wavelengths in the region of those required to pump the $6 s \rightarrow 7 s$ SERS transition [18]. This implies an absorption coefficient of $\sim 0.05 \mathrm{~cm}^{-1}$ at 10 torr, which is very considerable in view of the vapour column lengths of $\sim 25 \mathrm{~cm}$ which are used. However when using an intense focussed laser beam
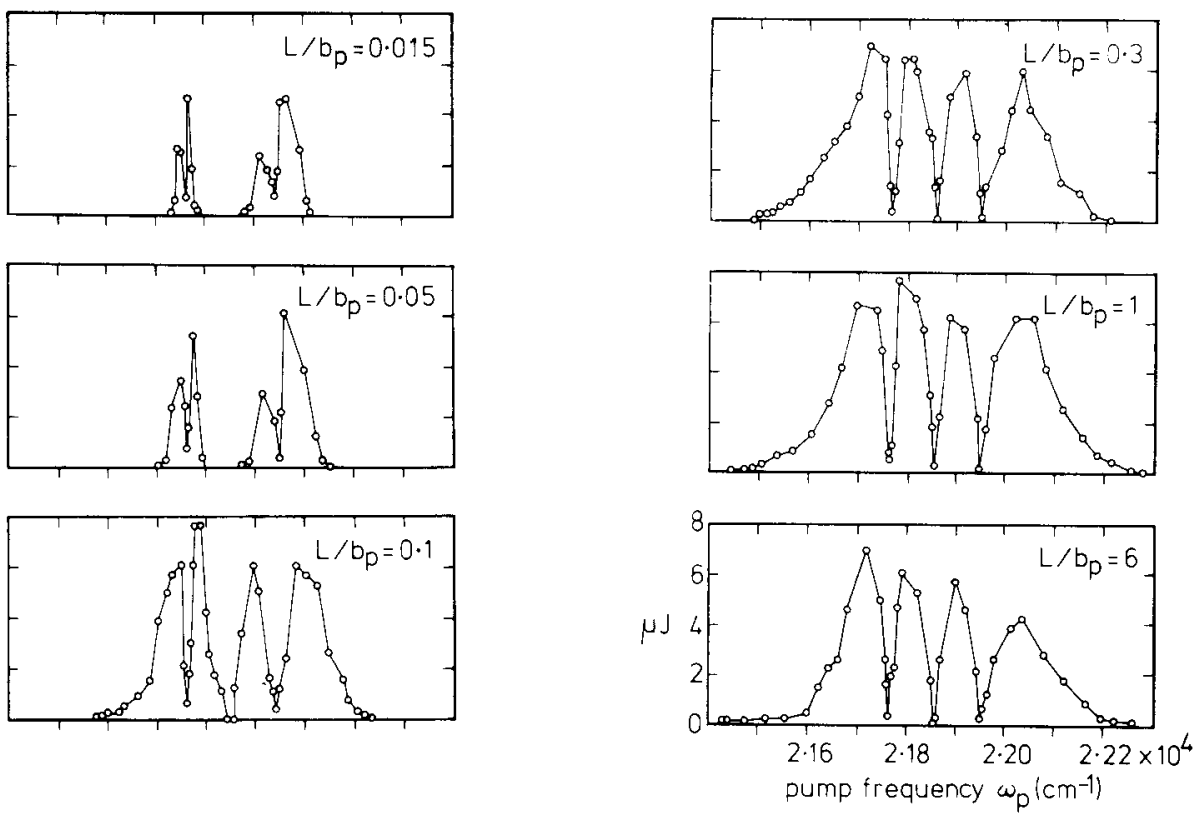

Figure 4 Infrared output energy as a function of dye laser tuning for different values of the pump beam focussing factor $L / b_{\mathbf{p}}$. 
this absorption can become saturated, and the total absorption loss decreases as the volume of the laser beam in the vapour is reduced. This volume is minimized when $L / b_{\mathrm{p}}=\sqrt{ } 3$. In that case less than $10 \%$ of a $140 \mu \mathrm{J}$ laser pulse is absorbed over a $25 \mathrm{~cm}$ vapour column at 10 torr.

In Fig. 4 it can be seen that a value for $L / b_{\mathrm{p}}$ of $\sim 0.3$ or greater is necessary to obtain continuous tuning between the $6 s-7 p_{1 / 2,3 / 2}$ doublet. As mentioned earlier, the best results in terms of tuning range and output energy are obtained with approximately confocal focussing of the pump beam $\left(L / b_{\mathrm{p}} \approx 1\right)$. By focussing more strongly $\left(L / b_{\mathrm{p}} \approx 6\right)$, the peak output energies are slightly lower and there is a more rapid fall off in the wings of the tuning curve.

Using confocal focussing, SERS tuning range measurements have been made with vapour pressures of 1, 3, 10 and 30 torr, and these are shown in Fig. 5. At 1 torr the effects of the inequality of the doublet oscillator strengths are very obvious. The ratios $f_{6 s-7 p_{3 / 2}} / f_{6 s-7 p_{1 / 2}}$ and $f_{7 s-7 p_{3 / 2}} /$ $f_{7 s-7 p_{1 / 2}}$ are each about 2 , and it can be clearly seen in Fig. 5 that this results in the tuning range around the $6 s-7 p_{3 / 2}$ resonance being nearly twice as wide as that around the $6 s-7 p_{1 / 2}$ resonance. At the same time the minimum Raman output energy with the pump tuned very close to the $p_{3 / 2}$ resonance is about half that obtained close to the $p_{1 / 2}$ resonance, while the width of the dip in output energy is also greater around the $p_{3 / 2}$ level. So whereas the tuning range is greater in the region of
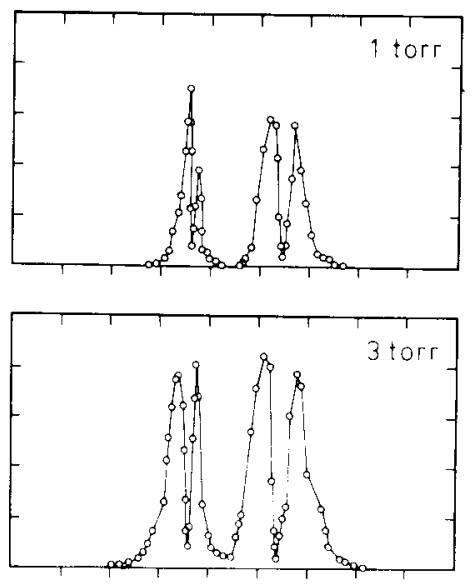

the stronger resonance, the effects of the resonant competing processes are also more pronounced.

The best performance has been obtained at 10 torr. Increasing the pressure to 30 torr has little effect on the tuning range and actually reduces the peak output energy by a factor of $\sim 2$. Evidence points to this being due to the greater absorption of the pump radiation by $\mathrm{Cs}_{2}$ molecules at the higher pressure [18].

With the dye laser beam focussed confocally $\left(L / b_{\mathrm{p}} \approx 1\right)$, the divergence of the generated SERS radiation, as measured by scanning a narrow slit across the beam in the far field, is 6-8 mrad (full angle to half maximum intensity points). This divergence angle does not appear to vary over the tuning range, and is in very good agreement with the calculated angle of $6 \mathrm{mrad}$ assuming that the infrared beam is diffraction-limited and emerges from a beam waist equal to that of the pump. We have also monitored the divergence of the residual dye laser radiation emerging from the heat pipe, and throughout the tuning range there is no evidence for any induced focussing or defocussing of the pump beam.

In stimulated Raman scattering, Stokes radiation travelling in both the forward and backward directions (parallel and antiparallel with the incident pump beam) is generated simultaneously. In the infrared source described here we have found that the maximum energy in the emergent backward Stokes wave is $\sim 2.5$ times smaller than that in the forward wave. This is in good agreement
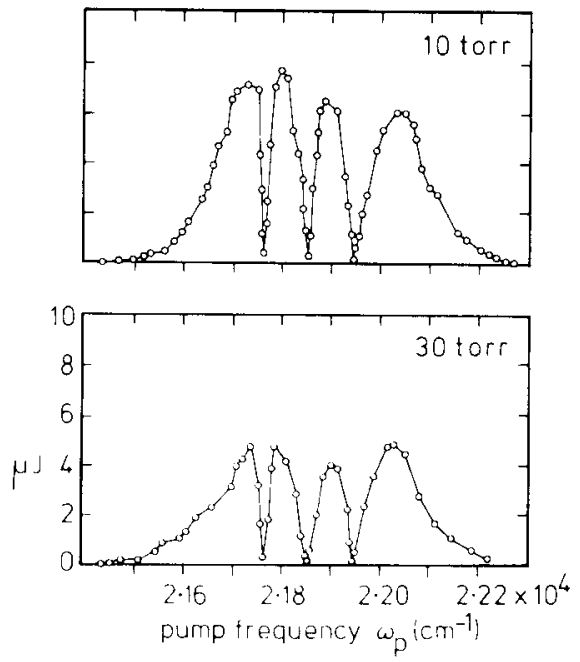

Figure 5 Infrared output energy as a function of dye laser tuning for different caesium vapour pressures. 
with the result of a numerical calculation of the saturation processes in SERS, which includes the dynamic competition between the forward and backward waves [19]. In addition it is observed that the useful ( $>50 \mathrm{~nJ}$ output) backward wave tuning range is $\sim 20 \%$ smaller.

The spectral linewidth of the generated Stokes radiation (in the forward direction) has been determined using a $1 \mathrm{~m} f / 10$ infrared grating monochromator (instrumental resolution $0.1 \mathrm{~cm}^{-1}$ ) and found to be in the range $0.25-0.55 \mathrm{~cm}^{-1}$. Within this range the linewidth is observed to increase as the vapour pressure is raised from 1 to 30 torr and also as the dye laser is tuned closer to the inter. mediate $7 p$ resonances, although the total variation amounts to no more than a factor of two in each case. These infrared linewidths are about an order of magnitude greater than the Doppler width of the two-photon Raman transition $\left(\sim 0.03 \mathrm{~cm}^{-1}\right)$ and also 2.5 times greater than the linewidth of the pump source $\left(0.1 \mathrm{~cm}^{-1}\right)$. In fact one may have expected the generated infrared linewidth to be somewhat narrower than the pump linewidth by virtue of gain narrowing. The fact that this is not the case suggests that some line-broadening processes are occurring. Our understanding of the factors influencing the SERS linewidth are incomplete at present and is the subject of continuing investigations.

Finally, we have studied the polarization of the Stokes radiation. Using a linearly polarized dye laser beam, over most of the tuning range the generated Stokes beam is also linearly polarized in the same direction. However for the range of pump frequencies between the $6 s-7 p_{1 / 2}$ resonance and a point nearly midway between the $7 p$ doublet, the infrared radiation emerges linearly polarized orthogonal to the pump polarization. A more detailed description of this behaviour and its explanation is given elsewhere [15].

The performance of this tunable infrared source can therefore be summarized briefly as in Table I.
TABLE I Summary of performance

\begin{tabular}{ll} 
Peak output energy & $8 \mu \mathrm{J}$ \\
Pulse duration & $5-6 \mathrm{~ns}$ \\
Peak power & $1.5 \mathrm{~kW}$ \\
Tuning range & $2.67-3.47 \mu \mathrm{m}^{\mathrm{a}}$ \\
Spectral width & $0.3-0.5 \mathrm{~cm}^{-1}$ \\
Output beam divergence & $6-8 \mathrm{mrad}$, diffraction-limited \\
Repetition rate & $4 \mathrm{~Hz}$ \\
Amplitude stability & $\pm 5 \% \mathrm{c}$ \\
& $\pm 80 \% \mathrm{~d}$ \\
\hline
\end{tabular}

a Continuously tunable over this $860 \mathrm{~cm}^{-1}$ range with $>50 \mathrm{~nJ}(10 \mathrm{~W})$ output.

b Limited by heating in the dye laser cell. Potentially could be increased to $50 \mathrm{~Hz}$ (maximum repetition rate of nitrogen laser) by incorporating a stirred or flowing dye solution.

c At centre of tuning range.

$d$ At edge of defined tuning range.

\section{Applications}

In order to demonstrate its simplicity of operation, the tunable source has been used to obtain infrared absorption spectra. The dual beam ratiometer arrangement shown in Fig. 6 was used. Frequency scanning was obtained by tuning the dye laser using a stepper motor drive to rotate the diffraction grating. Fig. 7 is an absorption spectrum of a sample of North Sea gas showing a part of the R-branch of the $\mathrm{CH}_{4}$ fundamental band around $3000 \mathrm{~cm}^{-1}$. This spectrum was obtained with a $4 \mathrm{~Hz}$ laser repetition rate, $\sim 3 \mathrm{~cm}^{-1} /$ minute frequency scanning rate and $3 \mathrm{~s}$ time constants in each detector channel. Thus the time required to scan the $80 \mathrm{~cm}^{-1}$ range shown in Fig. 7 was about 25 minutes. By increasing the repetition rate to $50 \mathrm{~Hz}$ (see Table $\mathrm{I}$ ), this scan rate could be reduced to 2 minutes.

Fig. 8 shows an absorption spectrum of ammonia gas, in which the principal lines are fundamental $\nu_{1}$ R-branch doublets. A large number of additional lines are visible particularly in

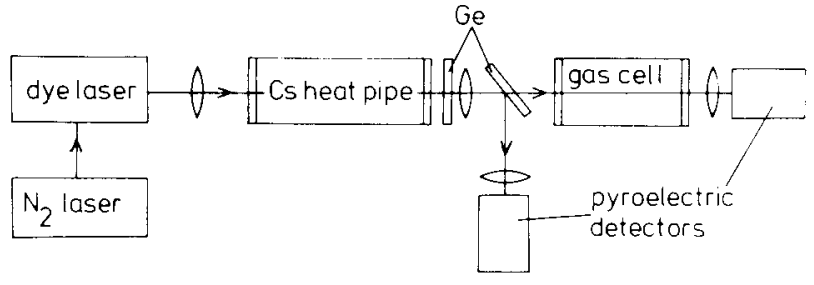

Figure 6 Dual beam spectrometer arrangement. The electrical signals from the two pyroelectric detectors are ratioed and the result displayed on a chart recorder. 


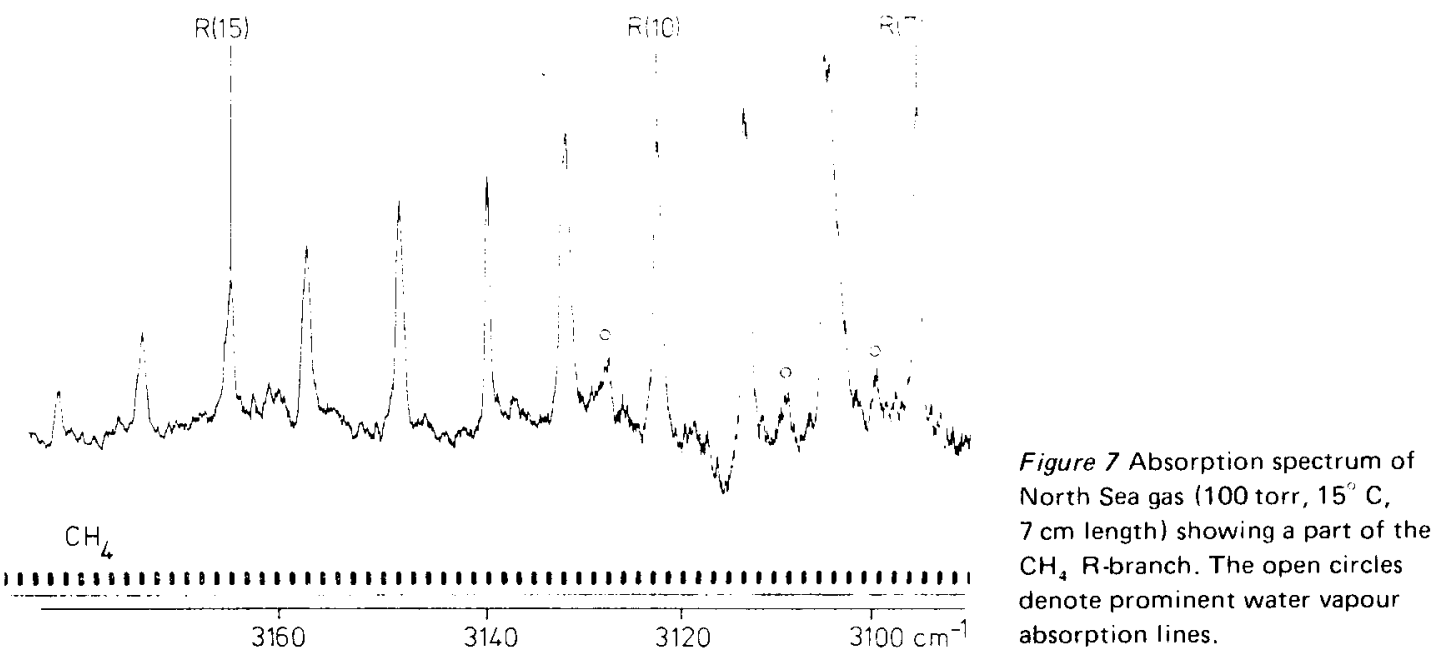

the region of the $\nu_{1} \mathrm{R}(4)$ doublet, and these are due to absorption by the overlapping fundamental $\nu_{3}$ band and also water vapour. The $\nu_{1} \mathrm{R}(5)$ lines provide a standard resolution test. These peaks are $1.8 \mathrm{~cm}^{-1}$ apart and numerical calculations shows that the degree of resolution shown in Fig. 8 implies an overall spectrometer resolution of $0.7 \mathrm{~cm}^{-1}$. This figure is within a factor of 2 of the measured SERS linewidth.

The observed resolution is similar to that available using a conventional grating monochromator. Thus, although the SERS source in its present form does not offer advantages of high resolution, we have at least shown that spectra comparable in quality to those obtained by conventional means can be obtained by using the SERS source in a very straightforward fashion. The advantages to be gained from using the SERS source lie in its brightness since this will allow

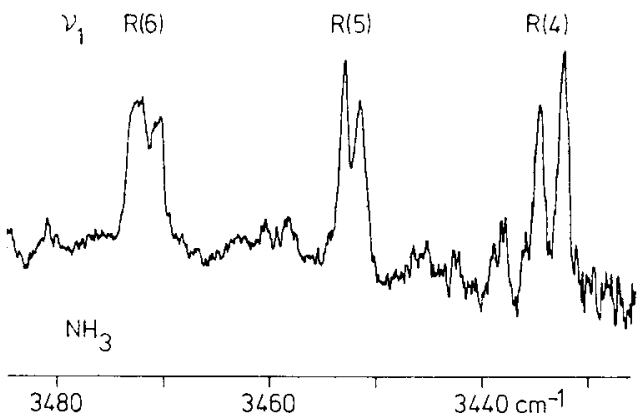

Figure 8 Absorption spectrum of ammonia gas (100 torr, $15^{\circ} \mathrm{C}, 7 \mathrm{~cm}$ length). The resolution of the $\nu_{1} R(5)$ doublet indicates an overall spectrometer resolution of $0.7 \mathrm{~cm}^{-1}$. spectra to be obtained in situations for which thermal sources are too weak (e.g. small samples, samples in inaccessible locations, etc.) Also the linewidths obtainable are certainly adequate for a number of applications involving selective vibrational excitation of molecular species. The short duration of the SERS pulse offers the further possibility of spectroscopic measurements with a time resolution of a few nanoseconds.

\section{Discussion}

We have described in some detail the construction and behaviour of a tunable infrared source based on SERS in caesium vapour. Compared with other tunable infrared lasers [1, 2], the SERS scheme offers outstanding advantages of simplicity in its construction and operation.

By using an atomic vapour as the nonlinear medium, many of the difficulties associated with crystals are avoided. There are no problems of commercial availability, poor optical quality or susceptibility to permanent laser-induced damage. At the same time a suitable heat pipe oven can be very easily built in the laboratory at low cost and without the need for special facilities or techniques. The reliability of the whole system should be determined largely by the reliability of the dye laser and its excitation source. An additional important feature of the SERS method is its scalability, making it attractive for high energy and power applications. By using a high power pump source, and by increasing the length and diameter of the heat pipe oven with a corresponding increase in beam 
size, much greater output energies are possible $[5,7]$.

Since the atomic vapour is used simply as a device for producing a fixed optical frequency shift, all the wavelength selection and tuning facilities are provided by the dye laser. Thus the extra infrared capability offered by SERS requires no additional wavelength control functions which would increase the complexity of an existing dye laser system. Frequency monitoring can, if desired, be carried out at the dye laser wavelengths. The infrared radiation emerges from the vapour colinearly with the dye laser beam, and this greatly simplifies the alignment of any optical components following the vapour cell. With SERS there are no critically controlled parameters and no phasematching or beam overlap adjustments are necessary. Results presented here show that the SERS output is relatively insensitive to the conditions of the alkali vapour, so there is no need for precise control of the temperature or buffer gas pressure. In addition there are no requirements for cavity alignment, fine angular adjustments, synchronous tuning of many optical components, cryogenics, or precision controlled magnetic fields or electric currents. These requirements add to the complexity and cost of other infrared sources.

Each one of the different types of infrared source now available has its own special merits and demerits which mean that it will not be the most effective or appropriate choice for every application. This is also true of SERS, which has two primary limitations. Firstly, the infrared linewidths that have been obtained at present $\left(0.3-0.5 \mathrm{~cm}^{-1}\right)$ are about an order of magnitude greater than those obtainable using pulsed semiconductor diode lasers and spin-flip Raman lasers, and 3-4 orders of magnitude greater than those obtained with $\mathrm{CW}$ semiconductor diode lasers, spin-flip Raman lasers and three-wave difference mixing. Our understanding of the factors influencing the SERS linewidths is incomplete at present and further work is required, but it is unlikely that in this respect at least SERS will ever compete with these other types of tunable lasers. Secondly it appears unlikely at present that complete coverage of the medium infrared range will be possible with SERS by using only one atomic vapour. However, even with these two limitations, the SERS source is still attractive for a great many spectroscopic applications.

In addition there are many opportunities for further development Table II contains a list of the electronic Raman transitions that can be reached using the fundamental frequencies of nitrogen laser-pumped dye lasers. Many more transitions can be reached by frequency-doubled dye laser outputs, and these include transitions

TABLE I I Summary of SERS transitions in alkali metal vapours accessible using the fundamental frequency of nitrogen laser-pumped dye lasers (Frequencies in $\mathrm{cm}^{-1}$ )

\begin{tabular}{|c|c|c|c|c|}
\hline $\begin{array}{l}\text { Alkali } \\
\text { metal }\end{array}$ & $\begin{array}{l}\text { SERS } \\
\text { transition } \\
\text { (Raman } \\
\text { shift in } \\
\text { brackets) }\end{array}$ & $\begin{array}{l}\text { Intermediate resonance } \\
\text { levels }\end{array}$ & $\begin{array}{l}\text { Corresponding } \\
\text { Stokes } \\
\text { frequencies }\end{array}$ & $\begin{array}{l}\text { Tuning } \\
\text { ranges } \\
\text { obtained }\end{array}$ \\
\hline$K^{19}$ & $\begin{array}{l}4 s \rightarrow 5 s \\
(21027)\end{array}$ & $\begin{array}{l}5 p_{1 / 2} 24701(404.8 \mathrm{~nm}) \\
5 p_{3 / 2} 24720(404.5 \mathrm{~nm})\end{array}$ & $\begin{array}{l}3675(2.72 \mu \mathrm{m}) \\
3693(2.71 \mu \mathrm{m})\end{array}$ & $2.56-3.5 \mu \mathrm{m}[6]$ \\
\hline $\mathrm{Rb}^{20}$ & $\begin{array}{l}5 s \rightarrow 6 s \\
(20134) \\
5 s \rightarrow 7 s \\
(26311)\end{array}$ & $\begin{array}{l}6 p_{1 / 2} 23715(421.7 \mathrm{~nm}) \\
6 p_{3 / 2} 23792(420.3 \mathrm{~nm}) \\
7 p_{1 / 2} 27835(359.3 \mathrm{~nm}) \\
7 p_{3 / 2} 27870(358.8 \mathrm{~nm})\end{array}$ & $\begin{array}{l}3582(2.79 \mu \mathrm{m}) \\
3659(2.73 \mu \mathrm{m}) \\
1524(6.56 \mu \mathrm{m}) \\
1559(6.42 \mu \mathrm{m})\end{array}$ & \\
\hline $\mathrm{Cs}^{21}$ & $\begin{array}{l}6 s \rightarrow 7 s \\
(18536) \\
6 s \rightarrow 8 s \\
(24317) \\
6 s \rightarrow 9 s \\
(26911)\end{array}$ & $\begin{array}{l}7 p_{1 / 2} 21766(459.4 \mathrm{~nm}) \\
7 p_{3 / 2} 21947(455.6 \mathrm{~nm}) \\
8 p_{1 / 2} 25709(389.0 \mathrm{~nm}) \\
8 p_{3 / 2} 25792(387.7 \mathrm{~nm}) \\
9 p_{1 / 2} 27637(361.8 \mathrm{~nm}) \\
9 p_{3 / 2} 27682(361.2 \mathrm{~nm})\end{array}$ & $\begin{array}{r}3230(3.09 \mu \mathrm{m}) \\
3411(2.93 \mu \mathrm{m}) \\
1392(7.18 \mu \mathrm{m}) \\
1475(7.78 \mu \mathrm{m}) \\
726(13.8 \mu \mathrm{m}) \\
771(13.0 \mu \mathrm{m})\end{array}$ & $\begin{array}{l}2.67-3.47 \mu \mathrm{m}^{\mathrm{a}} \\
2.5-4.75 \mu \mathrm{m}[6,7] \\
5.67-8.65 \mu \mathrm{m}[6] \\
11.7-15 \mu \mathrm{m}(6,7]\end{array}$ \\
\hline
\end{tabular}

a From Table I. 
to atomic Rydberg states which offer the opportunity of generating radiation over wide ranges of far infrared wavelengths. Future possibilities include the use of shorter wavelength dye lasers [23], and also the use of number of Raman transitions in alkaline earths which are as yet largely unexplored [5]. More sophisticated techniques could include the use of multi-pass and resonator configurations, although so far our preliminary experiments with a resonator have not proved very promising [24]. Finally it should be possible to considerably extend the range of infrared wavelengths by using transitions from previously populated excited states $[25,26]$.

However despite the many possibilities for greater sophistication, we believe that the simple basic source as described in this paper has a great deal to offer since it provides a very straightforward means of extending the wavelength coverage of existing dye laser systems.

\section{Acknowledgements}

Thanks are due to Drs T.R. Gilson and J. Black for valuable help with the construction of the heat pipe ovens, and to R. Baker for technical assistance with the dual beam ratiometer. This work has been supported by the Paul Instrument Fund and the Science Research Council. D. Cotter holds an 1851 Exhibition Research Fellowship.

\section{References}

1. M. J. COLLES and C. R. PIDGEON, Rep. Prog. Phys. 38 (1975) 329-460.

2. E. D. HIN KLEY, K. W. NILL and F. A. BLUM, in 'Laser Spectroscopy of Atoms and Molecules', edited by H. Walther (Springer-Verlag, Berlin, 1976), Topics in Applied Physics, 2.

3. F. P. SCHÄFER, (ed), 'Dye Lasers' (Springer-Verlag, Berlin, 1973), Topics in Applied Physics, 1.
4. P. P. SOROKIN, J. J. WYNNE and J. R. LANKARD, Appl. Phys. 22 (1973) 342-4.

5. J. L. CARLSTEN and P. C. I)UNN, Opt. Commun. 14 (1975) 8-12.

6. D. COTTFR, D. C. HANNA, P. A. KÄRKKÄINEN and R. WYATT, ibid 15 (1975) 143-6.

7. D. COTTER, D. C. HANNA and R. WYATT, ibid 16 (1976) $256-8$.

8. lor a review of stimulated Raman scattering and its applications, see M. MAIFR, Appl. Phys. 11 (1976) $209-31$.

9. C. R. VIDAl and J. COOPER, J. Appl. Phys. 40, (1969) 3370-4.

10. R. B. MILES and S. E. HARRIS, IEEE J. Quant. Elect. QE-9 (1973) 470-84.

11. Electrothermal Engineering Limited, 270 Neville Road, London E7 9QN; quartz fabric insulation heating tape HT550 $\left(800^{\circ} \mathrm{C} \max , 200 \mathrm{~W}\right)$.

12. Morganite Ceramic libres Limited, Neston, Wirral, Merseyside, England; Triton Kaowool fibre blanket.

13. T. W. HANSCH, Appl. Opt. 11 (1972) 895-8.

14. D. C. HANNA, P. A. KÄRKKÄINFN and R. WYATT, Opt. Quant. Elect. 7 (1975) $115-9$.

15. 1). COTTER and D. C. HANNA, J. Phys. B: Atom. Molec. Phys. 9 (1976) 2165-71.

16. J. J. WYNNE and P. P. SOROKIN, ibid. 8 (1975) L37-41.

17. D. COTTER, I). C. HANNA and R. WYATT, Appl. Phys. 8 (1975) 333-40.

18 M. LAPP and L. P. HARRIS, J. Quant Spectrosc. Radiat. Transfer 6 (1966) 169-79.

19. D. COTTER and D. C. HANNA, to be published (1977).

20. W. L. WIESE, M. W. SMITH and B. M. MILES, 'Atomic Transition Probabilities'. vol 2 (US GPO, Washington, DC, 1969), NSRDS-NBS Publication No. 22 .

21. C. E. MOORE, Atomic Energy Levels II, (US GPO, Washington, DC, 1952), US Dept of Commerce, NBS.

22. C. E. MOORE, ibid III, (1958).

23. C. RULLIERE, J. P. MORAND and O. de WITTE, Opt. Commun. 20 (1977) 339-41.

24. W.H.W. TUTTLEBEE, private communication.

25. M. ROKNI and S. YATSIV, Phys. Lett. 24A (1967) $277-8$.

26. R. T. V. KUNG and I. ITZKAN, Appl. Phys. Lett. 29 (1976) $780-3$. 